\title{
An inequality of Ostrowski-Grüss type for double integrals
}

Hüseyin Budak and Mehmet Zeki Sarıkaya

\begin{abstract}
In this study, we establish Ostrowski-Grüss type involving functions of two independent variables for double integrals. Cubature formula is also provided.
\end{abstract}

Mathematics Subject Classification (2010): 26D15.

Keywords: Ostrowski-Grüss type inequality, double integrals, two independent variables.

\section{Introduction}

In 1935, G. Grüss [7] proved the following inequality:

$$
\begin{aligned}
& \left|\frac{1}{b-a} \int_{a}^{b} f(x) g(x) d x-\frac{1}{b-a} \int_{a}^{b} f(x) d x \frac{1}{b-a} \int_{a}^{b} g(x) d x\right| \\
\leq & \frac{1}{4}\left(\Phi_{1}-\varphi_{1}\right)\left(\Phi_{2}-\varphi_{2}\right),
\end{aligned}
$$

provided that $f$ and $g$ are two integrable function on $[a, b]$ satisfying the condition

$$
\varphi_{1} \leq f(x) \leq \Phi_{1} \text { and } \varphi_{2} \leq g(x) \leq \Phi_{2} \text { for all } x \in[a, b] .
$$

The constant $\frac{1}{4}$ is best possible.

In 1938, Ostrowski established the following interesting integral inequality for differentiable mappings with bounded derivatives [9]:

Theorem 1.1 (Ostrowski inequality). Let $f:[a, b] \rightarrow R$ be a differentiable mapping on $(a, b)$ whose derivative $f^{\prime}:(a, b) \rightarrow R$ is bounded on $(a, b)$, i.e. $\left\|f^{\prime}\right\|_{\infty}:=$ $\sup \left|f^{\prime}(t)\right|<\infty$. Then, we have the inequality $t \in(a, b)$

$$
\left|f(x)-\frac{1}{b-a} \int_{a}^{b} f(t) d t\right| \leq\left[\frac{1}{4}+\frac{\left(x-\frac{a+b}{2}\right)^{2}}{(b-a)^{2}}\right](b-a)\left\|f^{\prime}\right\|_{\infty},
$$


for all $x \in[a, b]$. The constant $\frac{1}{4}$ is the best possible.

In 1882, P. L. Čebyšev [2] gave the following inequality:

$$
|T(f, g)| \leq \frac{1}{12}(b-a)^{2}\left\|f^{\prime}\right\|_{\infty}\left\|g^{\prime}\right\|_{\infty}
$$

where $f, g:[a, b] \rightarrow \mathbb{R}$ are absolutely continuous function, whose first derivatives $f^{\prime}$ and $g^{\prime}$ are bounded,

$$
\begin{aligned}
& T(f, g) \\
= & \frac{1}{b-a} \int_{a}^{b} f(x) g(x) d x-\left(\frac{1}{b-a} \int_{a}^{b} f(x) d x\right)\left(\frac{1}{b-a} \int_{a}^{b} g(x) d x\right)
\end{aligned}
$$

and $\|\cdot\|_{\infty}$ denotes the norm in $L_{\infty}[a, b]$ defined as $\|p\|_{\infty}=\underset{t \in[a, b]}{\operatorname{ess} \sup }|p(t)|$.

The following result of Grüss type was proved by Dragomir and Fedotov [4]:

Theorem 1.2. Let $f, u:[a, b] \rightarrow \mathbb{R}$ be such that $u$ is L-Lipshitzian on $[a, b]$, i.e,

$$
|u(x)-u(y)| \leq L|x-y| \text { for all } x \in[a, b],
$$

$f$ is Riemann integrable on $[a, b]$ and there exist the real numbers $m, M$ so that

$$
m \leq f(x) \leq M \text { for all } x \in[a, b] .
$$

Then we have the inequality,

$$
\left|\int_{a}^{b} f(x) d u(x)-\frac{u(b)-u(a)}{b-a} \int_{a}^{b} f(x) d x\right| \leq \frac{1}{2} L(M-m)(b-a) .
$$

From [8], if $f:[a, b] \rightarrow \mathbb{R}$ is differentiable on $(a, b)$ with the first derivative $f^{\prime}$ integrable on $[a, b]$, then Montgomery identity holds:

$$
f(x)=\frac{1}{b-a} \int_{a}^{b} f(t) d t+\int_{a}^{b} P(x, t) f^{\prime}(t) d t,
$$

where $P(x, t)$ is the Peano kernel defined by

$$
P(x, t)= \begin{cases}\frac{t-a}{b-a}, & a \leq t \leq x \\ \frac{t-b}{b-a}, & x<t \leq b .\end{cases}
$$

In [5], Dragomir and Wang proved following Ostrowski-Grüss type inequality using the inequality (1.1) and Montgomery identity (1.8):

Theorem 1.3. Let $f: I \subseteq \mathbb{R} \rightarrow \mathbb{R}$ be a differantiable mapping in $I^{\circ}$ and let $a, b \in I^{\circ}$ with $a<b$. If $f \in L_{1}[a, b]$ and

$$
\varphi_{3} \leq f^{\prime}(x) \leq \Phi_{3}, \forall x \in[a, b]
$$


then we have the following inequality

$$
\begin{aligned}
& \left|f(x)-\frac{1}{b-a} \int_{a}^{b} f(t) d t-\frac{f(b)-f(a)}{b-a}\left(x-\frac{a+b}{2}\right)\right| \\
\leq & \frac{1}{4}(b-a)\left(\Phi_{3}-\varphi_{3}\right),
\end{aligned}
$$

for all $x \in[a, b]$.

Barnett and Dragomir established following Ostrowski inequality for double integrals in [1]:

Theorem 1.4. Let $f:[a, b] \times[c, d] \rightarrow \mathbb{R}$ be a continuous on $[a, b] \times[c, d], f_{x y}=\frac{\partial^{2} f}{\partial x \partial y}$ exists on $(a, b) \times(c, d)$, and is bounded, i.e.,

$$
\left\|f_{x y}\right\|_{\infty}=\sup _{(x, y) \in(a, b) \times(c, d)}\left|\frac{\partial^{2} f(x, y)}{\partial x \partial y}\right|<\infty
$$

then we have the inequality

$$
\begin{aligned}
& \mid \int_{a}^{b} \int_{c}^{d} f(t, s) d s d t-\left[(b-a) \int_{c}^{d} f(x, s) d s\right. \\
& \left.+(d-c) \int_{a}^{b} f(t, y) d t-(b-a)(d-c) f(x, y)\right] \mid \\
& \leq \quad\left[\frac{1}{4}(b-a)^{2}+\left(x-\frac{a+b}{2}\right)^{2}\right]\left[\frac{1}{4}(d-c)^{2}+\left(y-\frac{c+d}{2}\right)^{2}\right]\left\|f_{x y}\right\|_{\infty}
\end{aligned}
$$

for all $(x, y) \in[a, b] \times[c, d]$.

In [1], the inequality (1.10) is established by the use of integral identity involving Peano kernels. In [10], Pachpatte obtained an inequality in the view (1.10) by using elementary analysis. The interested reader is also refered to $([1],[6],[10],[11],[13]-[15])$ for Ostrowski type inequalities in several independent variables.

Recently, Sarikaya and Kiris have proved the following Grüss type inequality for double integrals in [12]:

Theorem 1.5. Let $f, g:[a, b] \times[c, d] \rightarrow \mathbb{R}$ be two functions defined and integrable on $[a, b] \times[c, d]$. Then for

$$
\varphi \leq f(x, y) \leq \Phi \text { and } \gamma \leq g(x, y) \leq \Gamma \text { for all }(x, y) \in[a, b] \times[c, d]
$$


we have

$$
\begin{aligned}
& \mid \frac{1}{(b-a)(d-c)} \int_{a}^{b} \int_{c}^{d} f(x, y) g(x, y) d y d x \\
& \quad-\left(\frac{1}{(b-a)(d-c)} \int_{a}^{b} \int_{c}^{d} f(x, y) d y d x\right)\left(\frac{1}{(b-a)(d-c)} \int_{a}^{b} \int_{c}^{d} g(x, y) d y d x\right) \mid \\
& \leq \frac{1}{4}(\Phi-\varphi)(\Gamma-\gamma) .
\end{aligned}
$$

Moreover, Cerone and Dragomir [3] extended Gruss type inequalities for Lebesgue integrals on measurable spaces. This includes domaind from the plane provided in [12].

In this work, using the inequality (1.11), we will obtain an Ostrowski-Grüss type inequality for functions of two independent variables.

\section{Main results}

First, we give the following notations to simplify the presentation of some intervals.

$$
\begin{aligned}
& \Delta_{1}=[a, x] \times[c, y], \Delta_{2}=[a, x] \times[y, d], \\
& \Delta_{3}=[x, b] \times[c, y], \Delta_{4}=[x, b] \times[y, d] .
\end{aligned}
$$

Theorem 2.1. Let $f: \Delta:[a, b] \times[c, d] \rightarrow \mathbb{R}$ be a continuous on $\Delta, f_{x y}=\frac{\partial^{2} f}{\partial x \partial y}$ exists on $\Delta^{\circ}$. If $f$ integrable and

$$
\varphi \leq f_{x y}(x, y) \leq \Phi, \forall(x, y) \in \Delta
$$

then we have the following inequality

$$
\begin{aligned}
& \mid \frac{1}{(b-a)(d-c)} \int_{a}^{b} \int_{c}^{d} f(t, s) d s d t-\left[\frac{1}{(d-c)} \int_{c}^{d} f(x, s) d s\right. \\
& \left.\quad+\frac{1}{(b-a)} \int_{a}^{b} f(t, y) d t-f(x, y)\right] \\
& \quad-\frac{f(b, d)-f(b, c)-f(a, d)+f(a, c)}{(b-a)(d-c)}\left(x-\frac{a+b}{2}\right)\left(y-\frac{c+d}{2}\right) \mid \\
& \leq \frac{1}{4}(P-p)(\Phi-\varphi)
\end{aligned}
$$

where

$$
P=\max \{(x-a)(y-c),(b-x)(d-y)\}
$$


and

$$
p=\min \{(x-a)(y-d),(x-b)(y-c)\}
$$

for all $(x, y) \in \Delta$.

Proof. Define the kernel $p(x, t ; y, s)$ by

$$
p(x, t ; y, s):= \begin{cases}(t-a)(s-c), & \text { if }(t, s) \in[a, x] \times[c, y] \\ (t-a)(s-d), & \text { if }(t, s) \in[a, x] \times(y, d] \\ (t-b)(s-c), & \text { if }(t, s) \in(x, b] \times[c, y] \\ (t-b)(s-d), & \text { if }(t, s) \in(x, b] \times(y, d] .\end{cases}
$$

Then, we have

$$
\begin{aligned}
& \int_{a}^{b} \int_{c}^{d} p(x, t ; y, s) f_{t s}(t, s) d s d t \\
= & \int_{a}^{x} \int_{c}^{y}(t-a)(s-c) f_{t s}(t, s) d s d t+\int_{a}^{x} \int_{y}^{d}(t-a)(s-d) f_{t s}(t, s) d s d t \\
& +\int_{x}^{b} \int_{c}^{y}(t-b)(s-c) f_{t s}(t, s) d s d t+\int_{x}^{b} \int_{y}^{d}(t-b)(s-d) f_{t s}(t, s) d s d t \\
= & I_{1}+I_{2}+I_{3}+I_{4} .
\end{aligned}
$$

Let us calculate the integrals $I_{1}, I_{2}, I_{3}$ and $I_{4}$. Firstly, we have the equality

$$
\begin{aligned}
& I_{1}=\int_{a}^{x} \int_{c}^{y}(t-a)(s-c) f_{t s}(t, s) d s d t \\
& =\int_{a}^{x}(t-a)\left[(y-c) f_{t}(t, y)-\int_{c}^{y} f_{t}(t, s) d s\right] d t \\
& =(y-c) \int_{a}^{x}(t-a) f_{t}(t, y) d t-\int_{c}^{y}\left(\int_{a}^{x}(t-a) f_{t}(t, s) d t\right) d s \\
& =(y-c)\left[(x-a) f(x, y)-\int_{a}^{x} f(t, y) d t\right]-\int_{c}^{y}\left[(x-a) f(x, s)-\int_{a}^{x} f(t, s) d t\right] d s \\
& =(x-a)(y-c) f(x, y)-(y-c) \int_{a}^{x} f(t, y) d t-(x-a) \int_{c}^{y} f(x, s) d s+\int_{a}^{x} \int_{c}^{y} f(t, s) d s d t \text {. }
\end{aligned}
$$


Also, similar computations we have the equalities

$$
\begin{gathered}
I_{2}=\int_{a}^{x} \int_{y}^{d}(t-a)(s-d) f_{t s}(t, s) d s d t \\
=(x-a)(d-y) f(x, y)-(d-y) \int_{a}^{x} f(t, y) d t-(x-a) \int_{y}^{d} f(x, s) d s+\int_{a}^{x} \int_{y}^{d} f(t, s) d s d t \\
I_{3}=\int_{x}^{b} \int_{c}^{y}(t-b)(s-c) f_{t s}(t, s) d s d t \\
=(b-x)(y-c) f(x, y)-(y-c) \int_{x}^{b} f(t, y) d t-(b-x) \int_{c}^{y} f(x, s) d s+\int_{x}^{b} \int_{c}^{y} f(t, s) d s d t
\end{gathered}
$$

and

$$
I_{4}=\int_{x}^{b} \int_{y}^{d}(t-b)(s-d) f_{t s}(t, s) d s d t
$$

$=(b-x)(d-y) f(x, y)-(d-y) \int_{x}^{b} f(t, y) d t-(b-x) \int_{y}^{d} f(x, s) d s+\int_{x}^{b} \int_{y}^{d} f(t, s) d s d t$.

If we substitute the equalities (2.3)-(2.6) in (2.2), then we have

$$
\begin{gathered}
\int_{a}^{b} \int_{c}^{d} p(x, t ; y, s) f_{t s}(t, s) d s d t \\
=(b-a)(d-c) f(x, y)-(b-a) \int_{c}^{d} f(x, s) d s-(d-c) \int_{a}^{b} f(t, y) d t+\int_{a}^{b} \int_{c}^{d} f(t, s) d s d t .
\end{gathered}
$$

Applying Theorem 1.5 to mappings $p(x, . ; y,$.$) and f_{t s}(.,$.$) , we establish$

$$
\begin{aligned}
& \mid \frac{1}{(b-a)(d-c)} \int_{a}^{b} \int_{c}^{d} p(x, t ; y, s) f_{t s}(t, s) d s d t \\
& \quad-\left(\frac{1}{(b-a)(d-c)} \int_{a}^{b} \int_{c}^{d} p(x, t ; y, s) d s d t\right) \\
& \quad \times\left(\frac{1}{(b-a)(d-c)} \int_{a}^{b} \int_{c}^{d} f_{t s}(t, s) d s d t\right) \mid \\
& \leq \frac{1}{4}(\Phi-\varphi)(\Gamma-\gamma) .
\end{aligned}
$$


where

$$
\begin{aligned}
\Gamma= & \sup _{(t, s) \in \Delta} p(x, t ; y, s) \\
= & \max \left\{\sup _{(t, s) \in \Delta_{1}}(t-a)(s-c), \sup _{(t, s) \in \Delta_{2}}(t-a)(s-d),\right. \\
& \left.\sup _{(t, s) \in \Delta_{3}}(t-b)(s-c), \sup _{(t, s) \in \Delta_{4}}(t-b)(s-d)\right\} \\
= & \max \{(x-a)(y-c),(b-x)(d-y)\}=P,
\end{aligned}
$$

and

$$
\begin{aligned}
\gamma= & \inf _{(t, s) \in \Delta} p(x, t ; y, s) \\
= & \min \left\{\inf _{(t, s) \in \Delta_{1}}(t-a)(s-c), \inf _{(t, s) \in \Delta_{2}}(t-a)(s-d),\right. \\
& \left.\inf _{(t, s) \in \Delta_{3}}(t-b)(s-c), \inf _{(t, s) \in \Delta_{4}}(t-b)(s-d)\right\} \\
= & \min \{(x-a)(y-d),(x-b)(y-c)\}=p .
\end{aligned}
$$

Also, we have the equalities

$$
\begin{aligned}
& \int_{a}^{b} \int_{c}^{d} p(x, t ; y, s) d s d t \\
= & \int_{a}^{x} \int_{c}^{y}(t-a)(s-c) d s d t+\int_{a}^{x} \int_{y}^{d}(t-a)(s-d) d s d t \\
= & \frac{(x-a)^{2}(y-c)^{2}}{4}-\frac{(x-a)^{2}(d-y)^{2}}{4} \\
= & -\frac{(b-x)^{2}(y-c)^{2}}{4}+\frac{(b-x)^{2}(d-y)^{2}}{4} \\
= & {\left[(x-a)^{2}-(b-x)^{2}\right]\left[(y-c)^{2}-(d-y)^{2}\right] } \\
= & (b-a)(d-c)\left(x-\frac{a+b}{2}\right)^{2}\left(y-\frac{c+d}{2}\right) d s d t
\end{aligned}
$$


and

$$
\int_{a}^{b} \int_{c}^{d} f_{t s}(t, s) d s d t=f(b, d)-f(b, c)-f(a, d)+f(a, c) .
$$

If we put the equalities (2.7) and (2.9)-(2.12) in (2.8), then we obtain the desired inequality (2.1).

Corollary 2.2. With the assumptions in Theorem 2.1, if $\left|f_{x y}(x, y)\right| \leq M$ for all $(x, y) \in$ $[a, b] \times[c, d]$ and some positive constant $M$, then we have

$$
\begin{aligned}
& \mid \frac{1}{(b-a)(d-c)} \int_{a}^{b} \int_{c}^{d} f(t, s) d s d t \\
& -\left[\frac{1}{(d-c)} \int_{c}^{d} f(x, s) d s+\frac{1}{(b-a)} \int_{a}^{b} f(t, y) d t-f(x, y)\right] \\
& \leq-\frac{f(b, d)-f(b, c)-f(a, d)+f(a, c)}{(b-a)(d-c)}\left(x-\frac{a+b}{2}\right)\left(y-\frac{c+d}{2}\right) \mid \\
& \leq \frac{1}{2}(P-p) M
\end{aligned}
$$

where

$$
P=\max \{(x-a)(y-c),(b-x)(d-y)\}
$$

and

$$
p=\min \{(x-a)(y-d),(x-b)(y-c)\}
$$

for all $(x, y) \in[a, b] \times[c, d]$.

Corollary 2.3. Under assumptions of Theorem 2.1 with $x=\frac{a+b}{2}$ and $y=\frac{c+d}{2}$, we have the following inequality

$$
\begin{aligned}
& \mid \frac{1}{(b-a)(d-c)} \int_{a}^{b} \int_{c}^{d} f(t, s) d s d t-\left[\frac{1}{(d-c)} \int_{c}^{d} f\left(\frac{a+b}{2}, s\right) d s\right. \\
& \left.\quad+\frac{1}{(b-a)} \int_{a}^{b} f\left(t, \frac{c+d}{2}\right) d t-f\left(\frac{a+b}{2}, \frac{c+d}{2}\right)\right] \mid \\
& \leq \frac{1}{8}(b-a)(d-c)(\Phi-\varphi) .
\end{aligned}
$$


Corollary 2.4. Under assumption of Theorem 2.1 with $x=b$ and $y=d$, we get the inequality

$$
\begin{aligned}
& \mid \frac{1}{(b-a)(d-c)} \int_{a}^{b} \int_{c}^{d} f(t, s) d s d t \\
& -\left[\frac{1}{(d-c)} \int_{c}^{d} f(b, s) d s+\frac{1}{(b-a)} \int_{a}^{b} f(t, d) d t-f(b, d)\right] \\
& \quad-\frac{f(b, d)-f(b, c)-f(a, d)+f(a, c)}{4} \mid \\
& \leq \frac{1}{4}(b-a)(d-c)(\Phi-\varphi) .
\end{aligned}
$$

\section{Applications for cubature formulae}

Let us consider the arbitrary division $I_{n}: a=x_{0}<x_{1}<\ldots<x_{n}=b$, and $J_{m}: c=y_{0}<y_{1}<\ldots<y_{m}=d, h_{i}:=x_{i+1}-x_{i}(i=0, \ldots, n-1)$, and $l_{j}:=y_{j+1}-y_{j}$ $(j=0, \ldots, m-1)$,

$$
\begin{aligned}
& v(h):=\max \left\{h_{i} \mid i=0, \ldots, n-1\right\}, \\
& \mu(l):=\max \left\{l_{j} \mid j=0, \ldots, m-1\right\} .
\end{aligned}
$$

Then, the following theorem holds.

Theorem 3.1. Let $f:[a, b] \times[c, d] \rightarrow \mathbb{R}$ be as in Theorem 2.1 and $\xi_{i} \in\left[x_{i}, x_{i+1}\right]$ $(i=0, \ldots, n-1), \eta_{j} \in\left[y_{j}, y_{j+1}\right](j=0, \ldots, m-1)$ be intermediate points. Then we have the cubature formula:

$$
\begin{aligned}
& \int_{a}^{b} \int_{c}^{d} f(t, s) d s d t \\
= & \sum_{i=0}^{n-1} \sum_{j=0}^{m-1} h_{i} \int_{y_{j}}^{y_{j+1}} f\left(\xi_{i}, s\right) d s+\sum_{i=0}^{n-1} \sum_{j=0}^{m-1} l_{j} \int_{x_{i}}^{x_{i+1}} f\left(t, \eta_{j}\right) d t \\
& -\sum_{i=0}^{n-1} \sum_{j=0}^{m-1} h_{i} l_{j} f\left(\xi_{i}, \eta_{j}\right) \\
& +\sum_{i=0}^{n-1} \sum_{j=0}^{m-1}\left[f\left(x_{i+1}, y_{j+1}\right)-f\left(x_{i+1}, y_{j}\right)-f\left(x_{i}, y_{j+1}\right)+f\left(x_{i}, y_{j}\right)\right] \\
& \times\left(\xi_{i}-\frac{x_{i}+x_{i+1}}{2}\right)\left(\eta_{j}-\frac{y_{j}+y_{j+1}}{2}\right) \\
+ & R\left(\xi, \eta, I_{n}, J_{m}, f\right) .
\end{aligned}
$$


where the remainer term $R\left(\xi, \eta, I_{n}, J_{m}, f\right)$ satisfies the estimation

$$
\left|R\left(\xi, \eta, I_{n}, J_{m}, f\right)\right| \leq \frac{1}{4} v(h) \mu(l) \max _{i, j}\left(P_{i j}-p_{i j}\right)(\Phi-\varphi)
$$

where

$$
P_{i j}=\max \left\{\left(\xi_{i}-x_{i}\right)\left(\eta_{j}-y_{j}\right),\left(x_{i+1}-\xi_{i}\right)\left(y_{j+1}-\eta_{j}\right)\right\}
$$

and

$$
p_{i j}=\min \left\{\left(\xi_{i}-x_{i}\right)\left(\eta_{j}-y_{j+1}\right),\left(\xi_{i}-x_{i+1}\right)\left(\eta_{j}-y_{j}\right)\right\}
$$

Proof. Aplying Theorem 2.1 on the bidimentional interval $\left[x_{i}, x_{i+1}\right] \times\left[y_{j}, y_{j+1}\right]$, we get

$$
\begin{aligned}
& \mid \int_{x_{i}}^{x_{i+1}} \int_{y_{j}}^{y_{j+1}} f(t, s) d s d t \\
& -\left[h_{i} \int_{y_{j}}^{y_{j+1}} f\left(\xi_{i}, s\right) d s+l_{j} \int_{x_{i}}^{x_{i+1}} f\left(t, \eta_{j}\right) d t-h_{i} l_{j} f\left(\xi_{i}, \eta_{j}\right)\right] \\
& -\left[f\left(x_{i+1}, y_{j+1}\right)-f\left(x_{i+1}, y_{j}\right)-f\left(x_{i}, y_{j+1}\right)+f\left(x_{i}, y_{j}\right)\right] \\
& \quad \times\left(\xi_{i}-\frac{x_{i}+x_{i+1}}{2}\right)\left(\eta_{j}-\frac{y_{j}+y_{j+1}}{2}\right) \mid \\
& \leq \quad \frac{1}{4} h_{i} l_{j}\left(P_{i j}-p_{i j}\right)\left(\Phi_{i j}-\varphi_{i j}\right)
\end{aligned}
$$

where

$$
\Phi_{i j}:=\sup _{(t, s) \in\left[x_{i}, x_{i+1}\right] \times\left[y_{j}, y_{j+1}\right]}\left|f_{t s}(t, s)\right|, \quad \varphi_{i j}:=\inf _{(t, s) \in\left[x_{i}, x_{i+1}\right] \times\left[y_{j}, y_{j+1}\right]}\left|f_{t s}(t, s)\right|
$$

for all $i=0,1, \ldots, n-1 ; j=0,1, \ldots, m-1$.

Summing the inequality (3.3) over $i$ from 0 to $n-1$ and $j$ from 0 to $m-1$ and using the generalized triangle inequality, we get

$$
\begin{aligned}
\left|R\left(\xi, \eta, I_{n}, J_{m}, f\right)\right| & \leq \frac{1}{4} \sum_{i=0}^{n-1} \sum_{j=0}^{m-1} h_{i} l_{j}\left(P_{i j}-p_{i j}\right)\left(\Phi_{i j}-\varphi_{i j}\right) \\
& \leq \frac{1}{4} v(h) \mu(l) \max _{i, j}\left(P_{i j}-p_{i j}\right) \max _{i j}\left(\Phi_{i j}-\varphi_{i j}\right) \sum_{i=0}^{n-1} \sum_{j=0}^{m-1} 1 \\
& =\frac{n m}{4} v(h) \mu(l) \max _{i, j}\left(P_{i j}-p_{i j}\right)(\Phi-\varphi) .
\end{aligned}
$$

This completes the proof. 


\section{References}

[1] Barnett, N.S., Dragomir, S.S., An Ostrowski type inequality for double integrals and applications for cubature formulae, Soochow J. Math., 27(2001), no. 1, 109-114.

[2] Čebyšev, P.L., Sur less expressions approximatives des integrales definies par les autres prises entre les memes limites, Proc. Math. Soc. Charkov, 2(1882), 93-98.

[3] Cerone, P., Dragomir, S.S., A refinement of the Grüss inequality and applications, Tamkang J. Math., 38(2007), no. 1, 37-49.

[4] Dragomir, S.S., Fedotov, I., An inequality of Grüss type for Riemann-Stieltjes integral and applications for special means, Tamkang J. of Math., 29(1998), no. 4, 287-292.

[5] Dragomir, S.S., Wang, S., An inequality of Ostrowski-Grüss' type and its applications to the estimation of error bounds for some special means and for some sumerical quadrature rules, Computers Math. Applic., 33(1997), no. 11, 15-20.

[6] Dragomir, S.S., Barnett, N.S., Cerone, P., An n-dimensional version of Ostrowski's inequality for mappings of Hölder type, RGMIA Res. Pep. Coll., 2(1999), no. 2, 169-180.

[7] Grüss, G., Über das maximum des absoluten Betrages von $\frac{1}{b-a} \int_{a}^{b} f(x) g(x) d x-$ $\frac{1}{(b-a)^{2}} \int_{a}^{b} f(x) d x \int_{a}^{b} g(x) d x$, Math. Z., 39(1935), 215-226.

[8] Mitrinovic, D.S., Pecaric, J.E., Fink, A.M., Inequalities involving functions and their integrals and derivatives, Kluwer Academic Publishers, Dordrecht, 1991.

[9] Ostrowski, A.M., Über die absolutabweichung einer differentiebaren funktion von ihrem integralmitelwert, Comment. Math. Helv., 10(1938), 226-227.

[10] Pachpatte, B.G., On a new Ostrowski type inequality in two independent variables, Tamkang J. Math., 32(2001), no. 1, 45-49.

[11] Pachpatte, B.G., A new Ostrowski type inequality for double integrals, Soochow J. Math., 32(2006), no. 2, 317-322.

[12] Sarikaya, M.Z., Kiris, M.E., On Čebysev-Grüss type inequalities for double integrals, T.J.M.M., 7(2015), no. 1, 75-83.

[13] Sarikaya, M.Z., On the Ostrowski type integral inequality, Acta Math. Univ. Comenianae, 79(2010), no. 1, 129-134.

[14] Sarikaya, M.Z., On the Ostrowski type integral inequalityfor double integrals, Demostratio Mathematica, 45(2012), no. 3, 533-540.

[15] Ujević, N., Some double integral inequalities and applications, Appl. Math. E-Notes, 7(2007), 93-101.

Hüseyin Budak

Düzce University, Faculty of Science and Arts

Department of Mathematics

Konuralp Campus, Düzce, Turkey

e-mail: hsyn.budak@gmail.com

Mehmet Zeki Sarıkaya

Düzce University, Faculty of Science and Arts

Department of Mathematics

Konuralp Campus, Düzce, Turkey

e-mail: sarikayamz@gmail.com 